\title{
A importância da equipe NASF/AB - enfretamentos e multidisciplinariedade: uma revisão narrativa/crítica
}

\author{
The importance of the NASF/AB team - concerns and multidisciplinarity: a narrative/critical \\ review
}

La importancia del equipo NASF/AB - preocupaciones y multidisciplinariedad: una revisión narrativa/crítica

Weslley Barbosa Sales ${ }^{1 *}$, Allanna Stephany Cordeiro de Oliveira1, Luís Eduardo Alves Pereira1, Janine Greyce Martins de França ${ }^{1}$, Márcio Cavalcante Marcelino', Charlene Aguiar da Silva Gerônimo ${ }^{1}$, Amandha Eloisa Arcanjo Constantino', Rayza Brenda Tomaz Barbosa da Silva ${ }^{1}$, Rafaela Laissa Magalhães da Silva', Dimas Cícero Martins de França².

\section{RESUMO}

Objetivo: Identificar a importância no NASF-AB como estratégia de apoio, ampliação e inovação da atenção primária, destacando os principais enfrentamentos e sua rede multiprofissional. Revisão bibliográfica: Com a posição do Governo Federal, a partir da nota técnica, que diz respeito ao fim do cadastramento de novas equipes do NASF-AB, que ainda da liberdade ao gestor municipal e estadual de registrar os profissionais de saúde no Cadastro Nacional de Estabelecimentos de Saúde (CNES), observamos uma perda gigantesca no âmbito da Saúde. Considerações finais: Os profissionais que atuam no NASF-AB, têm desenvolvido ações de saúde como atividades domiciliares e ambulatoriais, atividades em grupo, atividades educativas em equipe. Corroborando com a importância do NASF-AB para o acesso integral a saúde, a literatura é clara no que se refere aos impactos que a presença dos profissionais do NASF-AB desempenha na qualidade de vida dos usuários do SUS, além de desenvolverem ações em educação em saúde e contribuírem na formação continuada em saúde.

Palavras-chave: Avaliação em saúde, Saúde da família, Atenção Primária à Saúde.

\begin{abstract}
Objective: To identify the importance in NASF-AB as a support strategy, expansion and innovation of primary care, highlighting the main challenges and its multiprofessional network. Bibliographic review: With the Federal Government's position, based on the technical note, which concerns the end of the registration of new NASF-AB teams, which still gives the municipal and state managers the freedom to register health professionals in the National Registry of Health Establishments (CNES), we observed a huge loss in the scope of Health. Final considerations: The professionals who work at NASF-AB, have developed health actions such as home and outpatient activities, group activities, educational activities in teams. Corroborating with the importance of NASF-AB for full access to health, the literature is clear with regard to the impacts that the presence of NASF-AB professionals has on the quality of life of SUS users, in addition to developing actions in education in health and contribute to continuing health education.
\end{abstract}

Keywords: Health assessment, Family health, Primary health care.

${ }^{1}$ Centro Universitário Maurício de Nassau, João Pessoa - PB. *E-mail: Weslleysaless8@gmail.com

${ }^{2}$ FPB - Faculdade Internacional da Paraíba, João Pessoa - PB. 


\section{RESUMEN}

Objetivo: identificar la importancia en NASF-AB como estrategia de apoyo, expansión e innovación de la atención primaria, destacando los principales desafíos y su red multiprofesional. Revisión bibliográfica: con la posición del Gobierno Federal, basada en la nota técnica, que se refiere al final del registro de nuevos equipos NASF-AB, que aún le da a los administradores municipales y estatales la libertad de inscribir profesionales de la salud en el Registro Nacional de Establecimientos de salud (CNES), observamos una gran pérdida en el alcance de la salud. Consideraciones finales: Los profesionales que trabajan en NASF-AB, han desarrollado acciones de salud tales como actividades domiciliarias y ambulatorias, actividades grupales, actividades educativas en equipos. Corroborando la importancia de NASF-AB para el pleno acceso a la salud, la literatura es clara con respecto a los impactos que la presencia de los profesionales de NASF-AB tiene en la calidad de vida de los usuarios del SUS, además de desarrollar la educación en salud.

Palabras clave: Evaluación de la salud, Salud familiar, Atención primaria de salud.

\section{INTRODUÇÃO}

O progresso do Sistema de Saúde brasileiro aconteceu de acordo com a evolução político econômica no Brasil, percorrendo regimes distintos e promulgações de Leis e Normas que atingiram diretamente a saúde pública até a criação do Sistema Único de Saúde (SUS) vigente (PAIVA CHA e TEIXEIRA LA, 2014). O SUS pode ser considerado uma das maiores conquistas sociais a contar da Constituição de 1988. Seus fundamentos apontam a democratização dos serviços da saúde e nas ações que passam a ser universalizados, ao invés de restritos, da mesma maneira que deixam de ser centralizados e mudam para nortear-se pela descentralização, como também a universalidade, equidade e integridade (PAIVA CHA e TEIXEIRA LA, 2014). Desde então muito se avançou dentre esses 30 anos, bem como testemunham os dados epidemiológicos sobre a distribuição de cobertura, acesso, infraestrutura de serviços, dentre vários outros. Contudo, estes avanços não foram contínuos, muito menos uniformes, como outros dados igualmente demonstram, tais como referentes à integralidade do acesso, equidade, financiamento e regulação do setor privado da saúde (VIACAVA F, et al., 2018).

Dentre os profissionais que podem fazer parte do NASF-AB, estão: Fisioterapeuta; farmacêutico; Fonoaudiólogo; Assistente social; nutricionista; profissional com formação em arte e educação (arte educador); psicólogo; terapeuta ocupacional; médico ginecologista/obstetra; médico homeopata; médico pediatra; médico veterinário; médico psiquiatra; médico geriatra; médico internista (clínica médica); médico do trabalho; médico acupunturista; e profissional de saúde sanitarista, ou seja, profissional graduado na área de saúde com pós-graduação em saúde pública ou coletiva ou graduado diretamente em uma dessas áreas (BRASIL, 2009; BRASIL, 2014; BRASIL, 2017). Ficando a critério da gestão mediante as necessidades do local em aderir os profissionais que mais se enquadrem dentro das necessidades do bairro ou município, ajustando os profissionais pela análise do território, atribuindo e organizando as suas tarefas com ações de promoção, prevenção e reabilitação (BRASIL, 2009; BRASIL, 2014; BRASIL, 2017).

Vemos que a atenção básica é porta de entrada para a saúde pública, onde milhões de pessoas recorrem a esses serviços em busca de profissionais que lhe recebam e atendam essas demandas de saúde, principalmente dos grupos de risco e das pessoas mais carentes. Entretanto, o que vivenciamos é o fim de mais um programa que atende a população de baixa renda, onde atualmente está em vigor uma nota técnica, que diz respeito a posição do atual governo federal, onde se deixa evidente através do documento que se dá fim ao cadastro de novas equipes NASF (Núcleo Ampliado as saúde da família) e desobriga e resguarda a gestão municipal/estadual de registrar novos profissionais no (CNES) Cadastro Nacional de Estabelecimentos de Saúde, instituído pelo Ministério da Saúde (PT/SAS 511/2000), que desse modo, não se há vinculo do profissional, tão pouco sobre a sua hora trabalhada. E havendo essa decisão de deixar a critério da gestão do município e do estado o registro desses profissionais, é evidente que muitos munícipios deixaram de adotar o NASF na atenção básica, assistiremos então a demissão em massa de diversos profissionais de saúde. 
Ademais, vemos a retirada desse programa que desde 2008 vem sendo trabalhada, e que não há modelos que possam substituir. Essa decisão do Governo Federal está intrinsicamente relacionada com o corte de verba que estão sendo realizadas tanto na saúde, quanto na educação, gerando grande turbulência nos pilares da educação e saúde pública no País (NEVES J, MACHADO K, 2019; OLIVEIRA J, 2020).

Sem dúvidas o Núcleo Ampliado de Saúde da Família e Atenção Básica (NASF-AB), possuí um papel primordial para a manutenção da saúde, visto que as demandas de saúde na rede de serviços da atenção primária, necessitam da participação de outros profissionais, proporcionando desse modo, resolutividade e abrangência dos serviços oferecidos pela atenção básica. Inquestionavelmente a desconstrução do NASF$A B$, acarretará grande retrocesso na saúde pública no País, visto que uma grande parcela da população é dependente desses serviços (GONÇALVES R, et al., 2015). Já se encontra batido, o que concerne a nossa Constituição Federal, que nos artigos 196 a 200, diz que " a Saúde é direito de todos e dever do estado ", Afirmando que é garantido políticas tanto sociais e econômicas para a conservação da saúde Brasileira, e que de tal maneira nos é assegurado o acesso universal, equânime e integral à esses serviços (SANTOS M, et al., 2017).

Sendo assim, levanta-se a seguinte problemática: Mediante todos esses enfretamentos que o NASF-AB vivencia atualmente, qual a importância desse programa para a saúde pública no País? Sendo o objetivo desse trabalho identificar e revisar na literatura a respeito da importância no NASF-AB como estratégia de apoio, ampliação e inovação da atenção primária, destacando os principais enfrentamentos e sua rede multiprofissional.

\section{REVISÃO BIBLIOGRÁFICA}

\section{Conhecimento sobre as políticas públicas de saúde e seus atuais enfretamentos}

Sabendo importância massiva que a rede de APS (Atenção Primária a Saúde) possui no Brasil, sendo este um país que possui uma alta incidência de doenças crônicas, o governo atual lançou uma nova proposta de financiamento para a APS. Para compreender as mudanças já vigentes, é preciso, de antemão, o conhecimento sobre o financiamento do antigo modelo e compreender a importância que uma rede de APS possui. Em 1997 foi criado o PAB (Piso da Atenção Básica) que é constituído por duas vertentes, o PAB fixo que chega a todas as 5.575 cidades do Brasil, e financia cada habitante com uma quantia que varia de $R \$ 23$ a R $\$ 28$ por habitante, com base nas características socioeconômicas de cada habitante, e o PAB variável que repassa verbas mensalmente baseando-se no número de equipes implantadas e composições profissionais previstas (NEVES J e MACHADO K, 2019).

Em 28 de setembro de 2017 foi criada a PNAB (Política Nacional de Atenção Básica), aprovada pela Portaria de Consolidação $n^{\circ} 2$ de 28 de setembro de 2017, a mesma constitui que o financiamento da APS seja tripartite e garantido nos Planos Nacional, Estadual e Municipal de gestão do Sistema Único de Saúde (SUS), já em dezembro do mesmo ano, a Portaria no 3.392 alterou as modalidades de transferência de recursos federais do SUS extinguindo os 5 blocos de financiamento (Atenção Básica, Vigilância em Saúde, Média e Alta Complexidade, Medicamentos e Gestão) e consolidando duas únicas formas de repasse: Custeio e Investimento (NEVES J e MACHADO K, 2019).

O novo modelo de financiamento apresentado na Portaria no. 2.979 de 12 de novembro de 2019 extingue o PAB fixo e variável e a adota a captação ponderada, como critério para o repasse dos recursos para custeio da atenção básica, é a revogação das equipes do Núcleo Ampliado de Saúde da Família e Atenção Básica (NASF-AB), sendo de responsabilidade dos gestores municipais a decisão de continuar, ou não, com a atuação do NASF. Além do repasse de verbas com base apenas nas pessoas cadastradas no SUS, existe a valorização do indivíduo "mais pobre", diferenciando cada indivíduo, principalmente, pela sua condição socioeconômica vulnerável, manchando diretamente um dos princípios do SUS, a universalidade, que garante o acesso universal ao sistema de saúde por parte de todos os indivíduos (OLIVEIRA J, 2020).

Há uma importância muito grande de haver outros profissionais integrados aos serviços da atenção Básica, visto que os programas que ainda existem ainda, são desproporcionais com a demanda de usuários do SUS, 
e com a inexistência do NASF gera como consequência a desassistência de milhares, além de claro o impacto na vida dos profissionais, uma vez que dia após dia perdem mais espaço no mercado de trabalho. A retomada do NASF é imprescindível no que diz respeito a saúde pública e de qualidade aqui no Brasil (OLIVEIRA J, 2020).

\section{A importância do fisioterapeuta no NASF-AB e seus desafios}

O fisioterapeuta é um profissional que faz parte do NASF-AB e que sua colaboração nos serviços de atenção básica à saúde é de extrema importância no que diz respeito a oferta de saúde (SOUZA DCM, et al., 2013). E por anos esse profissional foi visto apenas como reabilitador, desde o tempo do nascimento dessa profissão, esse pensamento por anos excluiu o fisioterapeuta das redes básicas de saúde, o que dificultava o acesso desse profissional a rede de saúde e como consequência acarretando uma grande dificuldade de acesso da população a esse serviço (RIBEIRO CD e FLORES-SOARES MC, 2015).

Atualmente, o fisioterapeuta segundo as diretrizes da (ESF) Estratégia da Saúde da Família, tem objetivos de desenvolver ações, promover serviços para tratamento e reabilitação, e trabalhar na prevenção de agravos e doenças da população, com ações de caráter individual, de grupos e da coletividade. E que deve atuar na Equipe Multiprofissional, através de uma abordagem interdisciplinar, objetivando a integralidade da assistência, explorando todo o arranjo da atenção básica (SOUZA D, et al., 2013; RIBEIRO CD e FLORESSOARES MC, 2015). A atuação deve ocorrer, preferencialmente, no contexto coletivo, com o envolvimento e a participação da população. Esta atuação de forma integrada na equipe possui como objetivo: planejar, implementar, controlar e executar políticas, programas, cursos, pesquisas ou eventos de saúde pública. Possui um grande potencial regulador, podendo funcionar como uma ligação entre a comunidade e a equipe na elaboração das ações de saúde (RODRIGUES F e BITENCOURT LTG, 2013).

Entretanto ainda existe muitas dificuldades nesse contexto principalmente no que concerne a utilização de ferramentas, equipamentos eletrônicos, recursos que são padrão ouro no atendimento individual, desde modo, grande parte das atividades exercidas são coletivas, tornando um pouco de lado a assistência individualizada, ademais a demanda é grande para poucos profissionais, o que podem gerar sobrecarga aos profissionais, sendo esse mais um motivo da permanência dos programas de ampliação a saúde, que geram a necessidade desses profissionais estarem inclusos como programas como o NASF. O fisioterapeuta desse modo, insere-se no sistema de atenção básica numa nova perspectiva de atuação gerencial, focada nas práticas de prevenção e promoção e não de uma forma exclusiva aos procedimentos de reabilitação, pelo contrário do que tem predominado nas ações deste segmento profissional (AVEIRO MC, et al., 2011).

\section{A importância do Profissional Fonoaudiólogo no NASF- AB}

As equipes do NASF são diversas, tendo a participação de muitos profissionais de saúde que integram uma rede ampliada, e que dentre esses professionais o fonoaudiólogo surge, integrando ações conjuntas com os demais profissionais de saúde, de modo a atender a população de acordo com os critérios e das análises que a gestão de saúde do local tenham realizado, dessa maneira existe o compartilhamento, apoio de toda a rede de profissionais, atuando na prevenção, promoção e reabilitação em saúde (SOLEMAN C e MARTINS CL, 2015).

A atuação do fonoaudiólogo na Atenção Básica encontra-se entre o campo clínico e o social, procedendo, um importante papel na rede de cuidados à saúde. Sabendo que o trabalho do fonoaudiólogo até pouco tempo atrás, firmava-se na média e alta complexidade e compreendendo a importância da contribuição deste profissional na atenção básica, identificou-se a necessidade de estimular essa atuação (FERNANDES T, et al., 2013).

Os fonoaudiólogos usualmente atuam na reabilitação com técnicas específicas para tratamentos individuais, qualificando encaminhamentos, orientando, compartilhando conhecimentos, contribuindo para maior resolubilidade dos casos de Fonoaudiologia, prevenindo e promovendo a saúde da comunicação. Sendo ações do fonoaudiólogo frente às demandas que lhe são encaminhadas pelas equipes de Saúde da Família, a promoção da linguagem oral e escrita, distúrbios articulatórios, voz, contação de histórias, memória, motricidade orofacial. Ademais na demanda específica do fonoaudiólogo do NASF, e que segundo Soleman 
C e Martins CL (2015), a mais recorrente é a que engloba as crianças com problemas no desenvolvimento de linguagem oral, escrita, e os distúrbios da leitura, que perpetuam para o atraso no desenvolvimento de fala e os distúrbios fonético/fonológicos. Encaminhadas por intermédio das equipes de Saúde da Família e escolas, além de queixas dos profissionais educadores trazidas pela mãe (SOLEMAN C e MARTINS CL, 2015).

Entretanto ainda existe uma baixa procura desse profissional no contexto da atenção básica, sendo esse um dos principais problemas causado pela falta de conhecimento sobre essa área que é essencial nos programas de atenção à saúde. Diversas são as ações citadas como possíveis intervenções, conforme apresenta o histórico vinculado a cada caso. Sendo realizadas desde consultas compartilhadas ou específicas para avaliação da criança, como também visitas domiciliares, com o intuito de uma melhor erudição do contexto familiar, e ainda visitas às escolas para a obtenção verdadeira da situação da criança (SOLEMAN C e MARTINS CL, 2015).

\section{A importância do Psicólogo para o NASF-AB}

A Organização Mundial de Saúde (OMS) conceitua a saúde como não apenas a ausência de doenças, mas sim um bem-estar biopsicossocial. Isto implica dizer que a saúde, hoje, é vista a partir de uma ótica integralizada, onde todas as esferas da vida do sujeito, a biológica, a psicológica e a social, se integram para que o bem-estar, lê-se saúde, seja atingido em sua totalidade. A partir desta forma de conceituação da saúde, as questões relativas ao psicológico e ao social do ser humano passaram a ganhar muito mais importância, ao ponto dos profissionais de saúde que trabalham com estas, como o psicólogo e o assistente social, serem introduzidos dentro da dinâmica de atendimento da Atenção Primaria a Saúde (APS) (ALVES R, et al., 2019).

No que diz respeito a saúde pública do Brasil, mais especificamente ao serviço de atendimento psicológico público, os Núcleos de Apoio à Saúde da Família (NASFs), possibilitaram que este serviço fosse feito a partir da inserção do profissional Psicólogo dentro das equipes multidisciplinares que formam e atuam através do programa. A portaria GM n. 154, 2008 preconiza que haja ao menos um Psicólogo em cada equipe. Sustentam ainda que a atuação do Psicólogo no NASF tem como base três propostas articuladas (Intervenções): Intervenções sanitárias, clínico-assistenciais e técnico-pedagógicas (ALVES RB, et al., 2019).

Através destas três intervenções bases do trabalho do Psicólogo dentro do NASF, percebe-se que este vai muito além do que somente a prática clínica. O Psicólogo se torna um agente provocador de mudanças biopsicossociais, fazendo um resgate da saúde da população atendida pelo programa em seus mais diversos âmbitos, se utilizando da Psicologia e seu arcabouço teórico-prático para tal, e seguindo as diretrizes do SUS e o código de ética vigente que norteia sua atuação profissional (ALVES RB, et al., 2019).

A cerca das ações de trabalho do Psicólogo, a partir das três intervenções acima descritas, podemos utilizar a sistematização destas ações desenvolvida por Nepomuceno LB e Brandão IR (2011), que listaram formas de atuações descritas de forma semelhante listadas pelos participantes de sua pesquisa, apesar desta listagem ter como base o trabalho do psicólogo na Estratégia Saúde da Família (ESF).

Tal sistematização trás as seguintes ações como formas de trabalho do Psicólogo na ESF (E consequentemente no NASF): Territorialização e planejamento local de saúde, Ações de acolhimento do centro de saúde da família, Visitas domiciliares, Ações de suporte à saúde mental, Ações de educação permanente junto à equipe de saúde da família e aos profissionais do território, Facilitação de grupo nas reuniões de equipes multiprofissionais, Ações comunitárias e de articulação de redes sociais, Consultoria a projetos sociais e Outras ações de fomento à participação e controle social. A partir de tais ações de trabalho do Psicólogo na ESF, em específico no NASF, as intervenções psicológicas ligam-se tanto à prevenção e à promoção da saúde como ao tratamento e à recuperação, vinculando-se à construção de soluções de curto, médio e longo prazo para os diversos dilemas de saúde da comunidade (NEPOMUCENO LB e BRANDAO IR, 2011).

Por fim, o trabalho do Psicólogo na saúde pública é de fundamental importância, na medida em que este consegue atuar em todas as três esferas que compõem o bem-estar humano: A biológica, a Psicológica e a Social. Porém, é preciso entender que este trabalho só chega a população através da ESF e principalmente através dos NASFs, pois estes possuem portaria específica de atuação do profissional Psicólogo, bem como 
o insere numa equipe multidisciplinar de atendimento, preenchendo as lacunas que a Psicologia possui no que se refere a outras áreas importantes da saúde, como a nutrição e a medicina propriamente dita. É imprescindível que os Núcleos de Apoio à Saúde da Família (NASFs) sejam mantidos pelo governo atual, só assim a população terá acesso ao que a Psicologia pode oferecer em prol de sua saúde mental (NEPOMUCENO LB e BRANDAO IR, 2011).

\section{A importância do Nutricionista como parte do NASF- AB}

O nutricionista é um profissional de suma importância na rede de atenção básica de saúde, pois, juntamente com a equipe multidisciplinar ele implementa diversas ações de promoção, tratamento e reabilitação da saúde. Porém, se tratando do impacto que a alimentação produz no desenvolvimento do indivíduo, tanto neurológico quanto físico, sua participação na Atenção Básica $(A B)$ ainda é um tanto reduzida. Considerando a transição epidemiológica e nutricional no Brasil, a última sendo caracterizada pela redução dos déficits nutricionais e aumento da ocorrência de sobrepeso e obesidade em todas as faixas etárias, mudanças vêm ocorrendo nas taxas de morbimortalidade, principalmente de doenças crônicas, tais como hipertensão arterial sistêmica (HAS) e diabetes mellitus, como também há alterações no estado nutricional da população em âmbito urbano e rural. A desigualdade socioeconômica também constitui um fator relevante, favorecendo em um extremo a desnutrição e em outro extremo a obesidade (GEUS LMM, et al., 2011).

As ações de nutrição e alimentação na atenção básica constituem uma ferramenta importantíssima pois previne às doenças e agravos não-transmissíveis, bem como auxiliam no desenvolvimento do indivíduo em diversas fases da sua vida, em especial durante a infância, na gestação e no período de amamentação. Sendo assim, torna-se indispensável ações em educação em saúde sobre a importância de uma alimentação saudável, com alimentos de todos os grupos alimentares e consultas com o profissional nutricionista para orientação sobre que tipo de dieta seguir (CERVATO-MANCUSO AM, et al., 2011)

\section{A importância do Profissional de Educação física na equipe NASF-AB}

A equipe de educação física atua diretamente na promoção de saúde, no qual, está conectada com o modo de vida dos indivíduos e das comunidades, assim como as mudanças advindas dessas realidades. Bem como, a promoção de saúde se remete a um maior número de estratégias e ações que objetivam aumentar e valorizar o potencial do indivíduo e da população a procura de um estilo de vida mais saudável (SAPORETTI G, et al., 2016).

As ações de promoção de saúde, acontece com diversos grupos, em destaque os: hipertensos, diabéticos, gestantes, adolescentes, tabagistas e idosos. Todas as ações são desenvolvidas por meio de estratégias diferentes, entre elas os exercícios e atividades físicas, as práticas corporais, as palestras, avaliação física, organização de eventos, intervenções em escolas e a formação de parcerias institucionais. Com isso, estás ações podem ser desenvolvidas no âmbito coletivo quanto no individual, ambos surtem bastante efeitos positivo na população (ROMERO A, et al., 2016)

Além disso, os educares físicos não atuam apenas na orientação e repasse de informações aos usuários para que adotassem hábitos mais saudáveis em seu cotidiano, mas também um acompanhamento contínuo, pois as mudanças só ocorrem na medida que os usuários se apropriam de seus benefícios e adotem o estilo de vida em seu dia a dia (SAPORETTI G, et al., 2016). Dessa forma fica evidente a importância do educador físico na equipe do NASF, principalmente pela presença de grupos de risco, os diabéticos, hipertensos, gestantes e idosos, que necessitam de acompanhamento e da realização de exercícios físicos (SAPORETTI G, et al., 2016).

\section{CONSIDERAÇÕES FINAIS}

O NASF-AB é uma equipe multidisciplinar, complementar que é composto por fisioterapeutas, terapeuta ocupacionais, nutricionistas, fonoaudiólogos, psicólogos, profissionais de educação física, entre outros, que ampliam a oferta de serviços e cuidados para a população, contribuindo com os pilares do SUS, principalmente no que concerne a integralização dos serviços, tratar o paciente como um todo. Com os atuais enfretamentos do NASF-AB, e com a criação da nova portaria, as verbas para a manutenção dessas equipes 
NASF-AB deixaram de existir, ficando a critério do município o vínculo com esses profissionais. Observa-se que as consequências disso são complexas, tornando o serviço de saúde pública no País cada vez mais obsoleto e retrógado.

\section{REFERÊNCIAS}

1. ALVES R, et al. "O Equilibrista": Atuação do Psicólogo no NASF no Vale do Itajaí. Psicologia e ciência, Brasília, 2019; 39:86-89.

2. AVEIRO M, et al. Perspectivas da participação do fisioterapeuta no Programa Saúde da Família na atenção à saúde do idoso. Ciência e saúde coletiva, Rio de Janeiro, 2011;16:1467-1478.

3. BRASIL, Ministério da Saúde. Secretaria de Atenção à Saúde. Cadernos da Atenção Básica: Diretrizes do NASF (Núcleo de Apoio a Saúde da Família). Brasília: Ministério da Saúde; 2009.

4. BRASIL, 2017, In: NÚCLEO de Apoio à Saúde da Família (NASF). Brasil: Estratégia Saúde da Família (ESF). Disponível em: https://www.saude.gov.br/acoes-e-programas/saude-da-familia/nucleo-de-apoio-a-saude-da-familianasf. Acesso em: 8 fev. 2020.

5. BRASIL. Ministério da Saúde. Secretaria de Atenção à Saúde. Caderno da Atenção Básica no 39 - Núcleo de Apoio à Saúde da Família - Volume 1: Ferramentas para a gestão e para o trabalho cotidiano. Brasília: Ministério da Saúde, 2014; 2:33-39.

6. FERNANDES T, et al. Análise das Atribuições dos Fonoaudiólogos do NASF em Municípios da Região Metropolitana do Recife. Revista CEFAC. 2013; 15:153-159.

7. GONÇALVES R, et al. Estudo do trabalho em Núcleos de Apoio à Saúde da Família (NASF), São Paulo, Brasil. Revista brasileira de saúde ocupacional. 2015; 40:59-74.

8. NEPOMUCENO LB, BRANDAO IR. Psicólogos na estratégia saúde da família: caminhos percorridos e desafios a superar. Psicologia e ciência. prof., 2011; 31(4): 762-777.

9. NEVES J, MACHADO K. 2019. In: ATENÇÃO primária à saúde terá novo modelo de financiamento. Brasil: EPSJV/Fiocruz. Disponível em: http://www.epsjv.fiocruz.br/noticias/reportagem/atencao-primaria-a-saude-tera-novomodelo-de-financiamento. Acesso em: 08 fev. 2020.

10. OLIVEIRA J. 2020. In: ESCLARECIMENTO sobre o novo modelo de financiamento da atenção primária à saúde. Brasil: Conselho Federal de Fonoaudiologia. Disponível em: https://www.fonoaudiologia.org.br/cffa/index.php/2020/01/esclarecimento-sobre-o-novo-modelo-de-financiamentoda-atencao-primaria-a-saude/. Acesso em: 08 fev. 2020.

11. OPAS/OMS. IN: Declaração de Alma-Ata. Conferência Internacional sobre Cuidados Primários em Saúde. 1978. Disponível em: http://www.opas.org.br.

12. PAIVA CHA, TEIXEIRA LA. Reforma sanitária e a criação do Sistema Único de Saúde: notas sobre contextos e autores. História, Ciências, Saúde - Manguinhos, Rio de Janeiro,2014; 21(1): 15-35.

13. RIBEIRO CD, FLORES-SOARES MC. Desafios para a inserção do fisioterapeuta na atenção básica: o olhar dos gestores. Revista de Saúde Pública, 2015;17(3): 379-393.

14. RODRIGUES F, BITENCOURT LTG. Atuação da Fisioterapia no Programa de Residência Multiprofissional. Revista do Programa de Residência Multiprofissional em Atenção Básica/Saúde da Família, 2013; 1(1): 46-53.

15. ROMERO A, et al. Interventions by Physical Education professionals in Family Health Support Units in São Paulo. Rev Bras Ativ Fís Saúde, 2016; 21:55-66.

16. RONZANI TM; RODRIGUES MC. O psicólogo na atenção primária à saúde: contribuições, desafios e redirecionamentos. Psicologia ciência. prof., 2006; 26(1):132-143.

17. SANTOS M, et al. Processo de Trabalho do Núcleo de apoio à Saúde da Família (NASF): Importância da Qualificação Profissional. Saúde \& Transformação Social / Health \& Social Change, 2017;8(2):60-69.

18. SANTOS NR. SUS 30 anos: o início, a caminhada e o rumo. Ciência \& Saúde Coletiva, 2018;23(6):1729-1736.

19. SAPORETTI G, et al. O profissional de educação física e a promoção da saúde em núcleos de apoio à saúde da família. Trab. Educ. Saúde, 2016; 14: 523-543.

20. SOLEMAN C, MARTINS CL. O trabalho do fonoaudiólogo no Núcleo de Apoio à Saúde da Família (NASF) especificidades do trabalho em equipe na atenção básica. Revista CEFAC, 2015; 17(4): 1241-1253.

21. SOUZA D, et al. Fisioterapia e Núcleo de Apoio à Saúde da Família: conhecimento, ferramentas e desafios. O Mundo da Saúde, São Paulo, 2013;37:176-184.

22. SOUZA M, et al. Integralidade na atenção à saúde: um olhar da equipe de saúde da família sobre a fisioterapia. $O$ Mundo da Saúde, São Paulo, 2012; 36:452-460.

23. VIACAVA F, et al. SUS: oferta, acesso e utilização de serviços de saúde nos últimos 30 anos. Ciência \& Saúde Coletiva, 2018;23:1751-1762.

24. GEUS LMM, et al. A importância da inserção do nutricionista na estratégia da saúde da família. Ciências \& Saúde Coletiva. Vol. 16 (supl.1):2011; 797-804.

25. CERVATO-MANCUSO AM, et al. A atuação do nutricionista na Atenção Básica à Saúde em um grande centro urbano. Ciência \& Saúde Coletiva, 17(12):2012; 3289-3300. 